\title{
Sharp Refinements for the Inverse Sine Function Related to Shafer-Fink's Inequality
}

\author{
Gabriel Bercu \\ Department of Mathematics and Computer Sciences, "Dunărea de Jos" University of Galaţi, 111 Domnească Street, \\ 800201 Galaţi, Romania \\ Correspondence should be addressed to Gabriel Bercu; gbercu@ugal.ro \\ Received 9 April 2017; Accepted 2 July 2017; Published 2 August 2017 \\ Academic Editor: Kishin Sadarangani \\ Copyright () 2017 Gabriel Bercu. This is an open access article distributed under the Creative Commons Attribution License, which \\ permits unrestricted use, distribution, and reproduction in any medium, provided the original work is properly cited.
}

The aim of our work is to give new sharp refinements of Shafer-Fink's inequality, using suitable changes of variables.

\section{Introduction and Motivation}

The inverse sine function is an elementary function that appears in many fields of engineering. In the communication theory and signal processing, it is used to describe the phase of a complex-valued signal. The inverse sine function also appears in the field of control theory, where a nonlinear network unit is modeled by a nonlinear function.

There are many applications in which the inverse sine must be replaced by an approximated function, for example, by a rational function. But we have to mention that finding a replacement of simple form for the inverse sine function is in fact difficult. That is why in our work we focus only on special analytic inequalities which have some interesting properties.

The starting point of this paper is Shafer-Fink's double inequality for the arc sine function:

$$
\frac{3 x}{2+\sqrt{1-x^{2}}} \leq \arcsin x \leq \frac{\pi x}{2+\sqrt{1-x^{2}}}, \quad 0 \leq x \leq 1 .
$$

Furthermore, 3 and $\pi$ are best constants in (1). Many refinements and extensions of this inequality have been provided (see, e.g., [1-9] and closely related references therein).

\section{Results and Discussion}

Since $\lim _{x \rightarrow 0}\left(\arcsin x-3 x /\left(2+\sqrt{1-x^{2}}\right)\right)=0$, it results that (1) gives a good results near zero, $\operatorname{arc} \sin x \approx 3 x /\left(2+\sqrt{1-x^{2}}\right)$ as $x \rightarrow 0$.
Related to the above approximation, we present the following inequality, which provides improvement of ShaferFink's inequality (1).

Theorem 1. For every real number $0 \leq x \leq 1$, the following two-sided inequality holds:

$$
\frac{x^{5}}{180}+\frac{x^{7}}{189} \leq \arcsin x-\frac{3 x}{2+\sqrt{1-x^{2}}} \leq \frac{\pi-3}{2} .
$$

Proof. The function

$$
\begin{aligned}
& f:[0,1] \longrightarrow \mathbb{R}, \\
& \qquad f(x)=\arcsin x-\frac{3 x}{2+\sqrt{1-x^{2}}}-\frac{x^{5}}{180}-\frac{x^{7}}{189}
\end{aligned}
$$

has the derivative

$$
f^{\prime}(x)=\frac{-4 x^{6}-3 x^{4}}{108}+\frac{-x^{2}+2-2 \sqrt{1-x^{2}}}{\sqrt{1-x^{2}}\left(\sqrt{1-x^{2}}+2\right)^{2}} .
$$

In order to prove that $f^{\prime}(x)>0$, for every $0 \leq x \leq 1$, we have to establish that

$$
\begin{aligned}
& 108\left(2-x^{2}-2 \sqrt{1-x^{2}}\right) \\
& \geq\left(4 x^{6}+3 x^{4}\right) \sqrt{1-x^{2}}\left(\sqrt{1-x^{2}}+2\right)^{2}
\end{aligned}
$$


or equivalently

$$
\begin{aligned}
& 16 x^{8}-4 x^{6}-12 x^{4}-108 x^{2}+216 \\
& \quad \geq \sqrt{1-x^{2}}\left(-4 x^{8}+17 x^{6}+15 x^{4}+216\right) .
\end{aligned}
$$

Since both sides of the above inequality are positive for all $0 \leq x \leq 1$, we can rise to the second power and obtain the following true result:

$$
\begin{aligned}
& x^{6}\left(16 x^{10}+104 x^{8}+177 x^{6}-27 x^{4}-5373 x^{2}+16767\right) \\
& \quad \geq 0 .
\end{aligned}
$$

We thus find that $f^{\prime}(x) \geq 0$, which imply that the function $f$ is strictly increasing on $[0,1]$.

For proving the right-hand side inequality from Theorem 1 , we introduce the function

$$
\begin{aligned}
& g:[0,1] \longrightarrow \mathbb{R}, \\
& \qquad g(x)=\arcsin x-\frac{3 x}{2+\sqrt{1-x^{2}}}-\frac{\pi-3}{2} .
\end{aligned}
$$

Its derivative $g^{\prime}(x)=\left(\sqrt{1-x^{2}}-1\right)^{2} / \sqrt{1-x^{2}}\left(\sqrt{1-x^{2}}+\right.$ $2)^{2}$ is positive for all $0 \leq x \leq 1$. Thus the function $g$ is strictly increasing on $[0,1]$ with $g(1)=0$, so $g(x) \leq 0$ on $[0,1]$.

The proof is completed.

Another way to extend the left-hand side inequality from (1) is to consider the approximation near the origin of the form $\arcsin x \approx(3 x+a(x)) /\left(1+2 \sqrt{1-x^{2}}\right)$, where $a(x) \rightarrow 0$ as $x \rightarrow 0$.

The result is stated as Theorem 2 .

Theorem 2. For every $0 \leq x \leq 1$, one has

$$
\arcsin x-\frac{3 x}{2+\sqrt{1-x^{2}}} \geq \frac{a(x)}{2+\sqrt{1-x^{2}}},
$$

where $a(x)=(1 / 60) x^{5}+(11 / 840) x^{7}$.

Proof. The function

$$
\begin{aligned}
h:[0,1] & \longrightarrow \mathbb{R}, \\
f(x)= & \arcsin x-\frac{3 x}{2+\sqrt{1-x^{2}}} \\
& -\frac{(1 / 60) x^{5}+(11 / 840) x^{7}}{2+\sqrt{1-x^{2}}}
\end{aligned}
$$

has the derivative

$$
h^{\prime}(x)=\frac{1}{\sqrt{1-x^{2}}}-\frac{\left(3+(1 / 12) x^{4}+(11 / 120) x^{6}\right)\left(2 \sqrt{1-x^{2}}+1-x^{2}\right)+3 x^{2}+(1 / 60) x^{6}+(11 / 840) x^{8}}{\sqrt{1-x^{2}}\left(2+\sqrt{1-x^{2}}\right)^{2}} .
$$
show

For proving that $h^{\prime}(x) \geq 0$ for all $0 \leq x \leq 1$, we have to

$$
\begin{gathered}
5-x^{2}+\frac{1}{840}\left(66 x^{8}-21 x^{6}-70 x^{4}-2520\right) \\
\geq 2 \sqrt{1-x^{2}}\left(1+\frac{1}{12} x^{4}+\frac{11}{120} x^{6}\right)
\end{gathered}
$$

or equivalently

$$
\begin{aligned}
& 66 x^{8}-21 x^{6}-70 x^{4}-840 x^{2}+1680 \\
& \geq 12 \sqrt{1-x^{2}}\left(11 x^{6}+10 x^{4}+120\right) .
\end{aligned}
$$

Since both sides of the above inequality are positive for all $0 \leq x \leq 1$, we can rise to the second power and deduce that

$$
\begin{aligned}
& x^{8}\left(4356 x^{8}+20944 x^{6}+10605 x^{4}-131460 x^{2}\right. \\
& \quad+759780) \geq 0
\end{aligned}
$$

which is true for every $0 \leq x \leq 1$.

The proof of Theorem 2 is completed.
In the following, we will discuss the right-hand side inequality from (1). More precisely, we state and prove the following results.

Theorem 3. For every $x \in[0,1]$ in the left-hand side and for every $x \in[0,0.871433]$ in the right-hand side, the following inequalities hold true:

$$
\begin{aligned}
& \left(1-\frac{\pi}{3}\right) x+\left(\frac{1}{6}-\frac{\pi}{18}\right) x^{3} \leq \arcsin x-\frac{\pi x}{2+\sqrt{1-x^{2}}} \\
& \quad \leq\left(1-\frac{\pi}{3}\right) x .
\end{aligned}
$$

Remark 4. Using MATLAB software, we found that the equation

$$
\arcsin x-\frac{\pi x}{2+\sqrt{1-x^{2}}}-\left(1-\frac{\pi}{3}\right) x=0
$$

has the real roots $x_{1}=0, x_{2,3} \approx \pm 0.871433$. 
We consider the function

$$
\begin{aligned}
p:[0,0.871433] & \longrightarrow \mathbb{R}, \\
p(x)= & \arcsin x-\frac{\pi x}{2+\sqrt{1-x^{2}}} \\
& -\left(1-\frac{\pi}{3}\right) x
\end{aligned}
$$

and its derivative

$$
\begin{aligned}
p^{\prime}(x)= & \frac{\left(5-x^{2}-\pi\right)+(4-2 \pi) \sqrt{1-x^{2}}}{\sqrt{1-x^{2}}\left(2+\sqrt{1-x^{2}}\right)^{2}} \\
& -\left(1-\frac{\pi}{3}\right) .
\end{aligned}
$$

We have to find the real number $\alpha \in[0,0.871433]$ so that $p^{\prime}(x) \leq 0$ for all $0 \leq x \leq \alpha$ or equivalently

$$
\begin{array}{r}
(9-4 \pi) x^{2}+\pi+3 \leq \sqrt{1-x^{2}}\left[(\pi-3) x^{2}+\pi+3\right], \\
0 \leq x \leq \alpha .
\end{array}
$$

Both sides of the above inequality are positive on $[0,0.871433]$, and hence we can rise to the second power and we find

$$
\begin{aligned}
& x^{2}\left[(\pi-3)^{2} x^{4}+\left(17 \pi^{2}-66 \pi+54\right) x^{2}\right. \\
& \left.+\left(-9 \pi^{2}+81\right)\right] \leq 0 .
\end{aligned}
$$

The polynomial function from the left-hand side has the real roots $x_{1}=x_{2}=0$ and $x_{3,4}=$ $\pm 3 \sqrt{2\left(\pi^{2}-9\right) /\left(54-66 \pi+17 \pi^{2}+(5 \pi-12) \sqrt{\pi(13 \pi-36)}\right)}$ $\approx 0.735975$.

We choose $\alpha=0.735975$.

Therefore, $p^{\prime}(x) \leq 0$ for all $0 \leq x \leq \alpha$ and $p^{\prime}(x)>0$ for all $\alpha<x \leq 0.871433$.

Since $p(0)=0$ and $p(0.871433)=0$, we obtain that $p(x) \leq 0$ on $[0,0.871433]$.

For proving the left-hand side inequality from Theorem 3 , we introduce the function

$$
\begin{aligned}
s:[0,1] & \longrightarrow \mathbb{R}, \\
p(x)= & \arcsin x-\frac{\pi x}{2+\sqrt{1-x^{2}}}-\left(1-\frac{\pi}{3}\right) x \\
& -\left(\frac{1}{6}-\frac{\pi}{18}\right) x^{3} .
\end{aligned}
$$

Its derivative is

$$
\begin{aligned}
s^{\prime}(x)= & \frac{\left(5-x^{2}-\pi\right)+(4-2 \pi) \sqrt{1-x^{2}}}{\sqrt{1-x^{2}}\left(2+\sqrt{1-x^{2}}\right)^{2}}-\left(1-\frac{\pi}{3}\right) \\
& -\left(\frac{1}{2}-\frac{\pi}{6}\right) x^{2} .
\end{aligned}
$$

The inequality $s^{\prime}(x) \geq 0$ on $[0,1]$ is equivalent to

$$
\begin{gathered}
\left(5-x^{2}-\pi\right)+(4-2 \pi) \sqrt{1-x^{2}} \\
\geq \sqrt{1-x^{2}}\left(5-x^{2}+4 \sqrt{1-x^{2}}\right) \\
\cdot\left[\left(1-\frac{\pi}{3}\right)+\left(\frac{1}{2}-\frac{\pi}{6}\right) x^{2}\right]
\end{gathered}
$$

or

$$
\begin{aligned}
& (12-4 \pi) x^{4}+(6-4 \pi) x^{2}+2 \pi+6 \\
& \quad \geq \sqrt{1-x^{2}}\left[(\pi-3) x^{4}+(9-3 \pi) x^{2}+2 \pi+6\right] .
\end{aligned}
$$

Both sides of the above inequality are positive on $[0,1]$; therefore we rise to the second power and we get the following true inequality on $[0,1]$ :

$$
\begin{aligned}
& x^{4}\left[(\pi-3)^{2} x^{6}+9(\pi-3) x^{4}\right. \\
& \left.\quad+3(\pi-3)(17 \pi-27) x^{2}+243+6 \pi-25 \pi^{2}\right] \geq 0 .
\end{aligned}
$$

In Theorem 5, we will state the following inequalities which give good results near the number 1 for the approximation $\arcsin x \approx \pi x /\left(2+\sqrt{1-x^{2}}\right)+b(x)$.

Theorem 5. For every $0 \leq x \leq 1$, one has

$$
\begin{aligned}
\frac{(\pi-4) \sqrt{1-x}}{2 \sqrt{2}} & \leq \arcsin x-\frac{\pi x}{2+\sqrt{1-x^{2}}} \\
& \leq \frac{(\pi-4) \sqrt{1-x}}{2 \sqrt{2}}+\frac{\pi(1-x)}{4} .
\end{aligned}
$$

Remark 6. In order to improve the right-hand side of ShaferFink's inequality (1), we impose that $(\pi-4) \sqrt{1-x} / 2 \sqrt{2}+\pi(1-$ $x) / 4 \leq 0$, which is true for $x \in[\beta, 1]$, where $\beta=\left(-\pi^{2}+16 \pi-\right.$ $32) / \pi^{2} \approx 0.85068$.

Proof. We consider the function

$$
\begin{aligned}
& r:[0,1] \longrightarrow \mathbb{R}, \\
& r(x)=\arcsin x-\frac{\pi x}{2+\sqrt{1-x^{2}}}-\frac{(\pi-4) \sqrt{1-x}}{2 \sqrt{2}}
\end{aligned}
$$

and its derivative

$$
\begin{aligned}
r^{\prime}(x)= & \frac{\left(5-x^{2}-\pi\right)+(4-2 \pi) \sqrt{1-x^{2}}}{\sqrt{1-x^{2}}\left(2+\sqrt{1-x^{2}}\right)^{2}} \\
& +\frac{\pi-4}{4 \sqrt{2} \sqrt{1-x}} .
\end{aligned}
$$

We note that $r(1)=0$. In order to obtain $r(x) \geq 0$ on $[0,1]$, we have to prove that $r^{\prime}(x) \leq 0$ for all $x \in[0,1]$ or equivalently

$$
\begin{array}{r}
4 \sqrt{2}\left[5-\pi-x^{2}+(4-2 \pi) \sqrt{1-x^{2}}\right] \\
\leq(4-\pi) \sqrt{1+x}\left(2+\sqrt{1-x^{2}}\right)^{2} .
\end{array}
$$


The real roots of the function from the left-hand side of (29) are

$$
\begin{aligned}
x_{1,2} & = \pm \sqrt{-3+7 \pi-2 \pi^{2}+2(-2+\pi) \sqrt{(-3+\pi) \pi}} \\
& \approx \pm 0.8801802 .
\end{aligned}
$$

Case 1. For all $0 \leq x \leq 0.8801802$, the function from the left-hand side of (29) is nonpositive and the function from the right-hand side of (29) is positive; hence inequality (29) holds true.

Case 2. For $0.8801802<x \leq 1$, both sides of inequality (29) are positive, and thus we can rise to the second power and obtain

$$
\begin{aligned}
& 8 \sqrt{1-x^{2}}\left[(\pi-4)^{2} x^{3}+\left(-16+8 \pi+\pi^{2}\right) x^{2}\right. \\
& \left.-5(\pi-4)^{2} x+11 \pi^{2}-72 \pi+80\right] \leq(\pi-4)^{2} x^{5} \\
& -\left(16+8 \pi-\pi^{2}\right) x^{4}-26(\pi-4)^{2} x^{3}+2(208 \\
& \left.-184 \pi+51 \pi^{2}\right) x^{2}+41(\pi-4)^{2} x-119 \pi^{2}+504 \pi \\
& -656 .
\end{aligned}
$$

The polynomial function $R(x)=(\pi-4)^{2} x^{3}+(-16+8 \pi+$ $\left.\pi^{2}\right) x^{2}-5(\pi-4)^{2} x+11 \pi^{2}-72 \pi+80$ has the real roots $x_{1} \approx$ $-25.9051, x_{2} \approx 1.3467$, or $x_{3} \approx 1.4637$; hence $R(x)<0$ for all $0.8801802<x \leq 1$.

The polynomial function $Q(x)=(\pi-4)^{2} x^{5}-(16+8 \pi-$ $\left.\pi^{2}\right) x^{4}-26(\pi-4)^{2} x^{3}+2\left(208-184 \pi+51 \pi^{2}\right) x^{2}+41(\pi-$ $4)^{2} x-119 \pi^{2}+504 \pi-656$ has the real roots $x_{1} \approx 2.9092$, $x_{2} \approx-1.0537, x_{3}=1, x_{4} \approx 2.5536$, or $x_{5} \approx 42.8403$; hence $\mathrm{Q}(x)<0$ for all $0.88018026<x \leq 1$.

Therefore, both sides of inequality (31) are nonpositive, so we multiply it by $(-1)$ and then we can rise to the second power and obtain

$$
(1-x) \cdot S(x) \geq 0
$$

where

$$
\begin{aligned}
S(x) & =\left(\pi^{4}-16 \pi^{3}+96 \pi^{2}-256 \pi+256\right) x^{9}+\left(3 \pi^{4}\right. \\
& \left.-48 \pi^{3}+224 \pi^{2}-256 \pi-256\right) x^{8}+\left(16 \pi^{4}\right. \\
& \left.-256 \pi^{3}+1408 \pi^{2}-3072 \pi+3072\right) x^{7}+\left(296 \pi^{4}\right. \\
& \left.-1792 \pi^{3}-128 \pi^{2}+11264 \pi-3072\right) x^{6}+\left(618 \pi^{4}\right. \\
& \left.-4000 \pi^{3}+10560 \pi^{2}-13824 \pi+13824\right) x^{5} \\
& +\left(-4202 \pi^{4}+38688 \pi^{3}-101312 \pi^{2}+66048 \pi\right. \\
& -13824) x^{4}+\left(7416 \pi^{4}-34176 \pi^{3}+35712 \pi^{2}\right. \\
& -27648 \pi+27648) x^{3}+\left(14160 \pi^{4}-84096 \pi^{3}\right. \\
& \left.+107904 \pi^{2}+64512 \pi-27648\right) x^{2}+\left(-3699 \pi^{4}\right. \\
+ & \left.1584 \pi^{3}+42336 \pi^{2}-20736 \pi+20736\right) x
\end{aligned}
$$

$$
\begin{aligned}
& +\left(-6417 \pi^{4}+18576 \pi^{3}+34272 \pi^{2}-76032 \pi\right. \\
& -20736) .
\end{aligned}
$$

Using MATLAB software, we find that the polynomial function $S$ has only one real root $x_{1} \approx-0.9411$; hence (1$x) \cdot S(x) \geq 0$ on $[0.88018026,1]$.

For proving the right-hand side of the inequality from Theorem 5 we introduce the function

$$
\begin{aligned}
t:[0,1] & \longrightarrow \mathbb{R}, \\
t(x)= & \arcsin x-\frac{\pi x}{2+\sqrt{1-x^{2}}}-\frac{(\pi-4) \sqrt{1-x}}{2 \sqrt{2}} \\
& -\frac{\pi(1-x)}{4} .
\end{aligned}
$$

We note that $t(1)=0$; therefore in order to demonstrate the inequality $t(x) \leq 0$ on $[0,1]$, we have to establish that $t^{\prime}(x) \geq 0$ on $[0,1]$ or equivalently

$$
\begin{aligned}
2 \sqrt{2} & -4 \sqrt{2}(1+\pi) x^{2} \\
& +\left(16 \sqrt{2}-3 \pi \sqrt{2}-\pi \sqrt{2} \cdot x^{2}\right) \sqrt{1-x^{2}} \\
\geq & (4-\pi)\left[4(x+1) \sqrt{1-x}-\left(x^{2}-5\right) \sqrt{1+x}\right] .
\end{aligned}
$$

Since both sides of the above inequality are positive on $[0,1]$, we can rise to the second power and get

$$
(x-1)\left[8 \sqrt{1-x^{2}} \cdot U(x)-V(x)\right] \geq 0,
$$

where

$$
\begin{aligned}
U(x)= & \left(2 \pi^{2}+2 \pi\right) x^{3}+\left(3 \pi^{2}-6 \pi+16\right) x^{2} \\
& +\left(10 \pi^{2}-50 \pi\right) x+\left(5 \pi^{2}-10 \pi-80\right) \\
V(x)= & 2 \pi^{2} x^{5}+\left(16-8 \pi+3 \pi^{2}\right) x^{4} \\
& -18\left(\pi^{2}+8 \pi\right) x^{3} \\
& +\left(-416+64 \pi-44 \pi^{2}\right) x^{2} \\
& +\left(464 \pi-64 \pi^{2}\right) x+656+136 \pi-23 \pi^{2}
\end{aligned}
$$

The polynomial function $U(x)$ has the real roots $x_{1} \approx$ $1.43, x_{2} \approx-1.10$, and $x_{3} \approx 1.5081$; hence $U(x)<0$ on $[0,1]$.

The polynomial function $V(x)$ has the real roots $x_{1} \approx$ $-5.53, x_{2} \approx-1.13, x_{3} \approx-1.0713, x_{4} \approx 1.1722$, and $x_{5} \approx 5.52$; hence $V(x)>0$ on $[0,1]$.

Therefore, inequality $(36)$ is true on $[0,1]$.

This completes the proof. 
Remark 7. Combining the results of Theorems 3 and 5, respectively, we note that we improve the right-hand side of Shafer-Fink's inequality on $[0,1]$ as follows:

$$
\begin{array}{r}
\arcsin x \leq \frac{\pi x}{2+\sqrt{1-x^{2}}}+\left(1-\frac{\pi}{3}\right) x \\
\forall 0 \leq x \leq 0.871433, \\
\arcsin x \leq \frac{\pi x}{2+\sqrt{1-x^{2}}}+\frac{(\pi-4) \sqrt{1-x}}{2 \sqrt{2}}+\frac{\pi(1-x)}{4} \\
\forall 0.85068 \leq x \leq 1 .
\end{array}
$$

\section{Conclusion}

In the present work we investigated the approximation of the inverse sine function and obtained new bounds. We have deduced lower and upper bounds which are sharp and very accurate and also improve Shafer-Fink's inequality.

\section{Conflicts of Interest}

The author declares that he has no conflicts of interest.

\section{References}

[1] B.-N. Guo and F. Qi, "Sharpening and generalizations of Carlson's inequality for the arc cosine function," Hacettepe Journal of Mathematics and Statistics, vol. 39, no. 3, pp. 403-409, 2010.

[2] B. J. Malešević, "An application of $\lambda$-method on Shafer-Fink's inequality," Univerzitet u Beogradu. Publikacije Elektrotehničkog Fakulteta. Serija Matematika, vol. 8, pp. 90-92, 1997.

[3] B. J. Malešević, "An application of $\lambda$-method on inequalities of Shafer-Fink-type," Mathematical Inequalities \& Applications, vol. 10, no. 3, pp. 529-534, 2007.

[4] D. S. Mitrinović, Analytic Inequalities, Springer, Berlin, Germany, 1970.

[5] C. Mortici and H. M. Srivastava, "Estimates for the arctangent function related to Shafer's inequality," Colloquium Mathematicum, vol. 136, no. 2, pp. 263-270, 2014.

[6] Y. Hu and C. Mortici, "A lower bound on the sinc function and its application," The Scientific World Journal, vol. 2014, Article ID 571218, 4 pages, 2014.

[7] F. Qi and B.-N. Guo, "Sharpening and generalizations of Shafer's inequality for the arc sine function," Integral Transforms and Special Functions. An International Journal, vol. 23, no. 2, pp. 129-134, 2012.

[8] L. Zhu, "On Shafer-Fink inequalities," Mathematical Inequalities \& Applications, vol. 8, no. 4, pp. 571-574, 2005.

[9] L. Zhu, "On Shafer-Fink-type inequality," Journal of Inequalities and Applications, vol. 2007, Article ID 67430, 4 pages, 2007. 


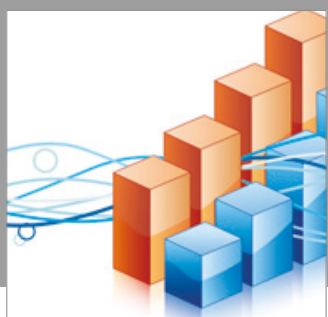

Advances in

Operations Research

vatersals

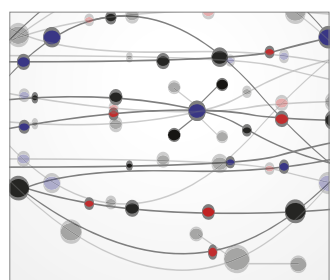

\section{The Scientific} World Journal
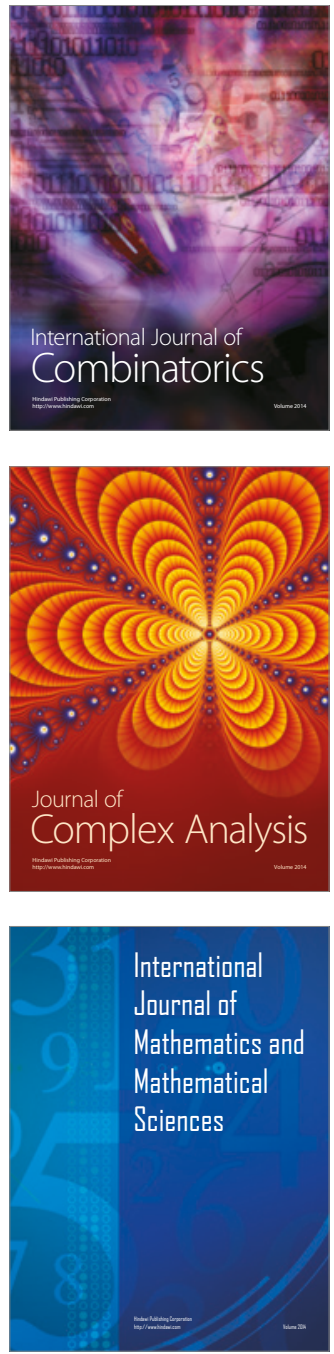
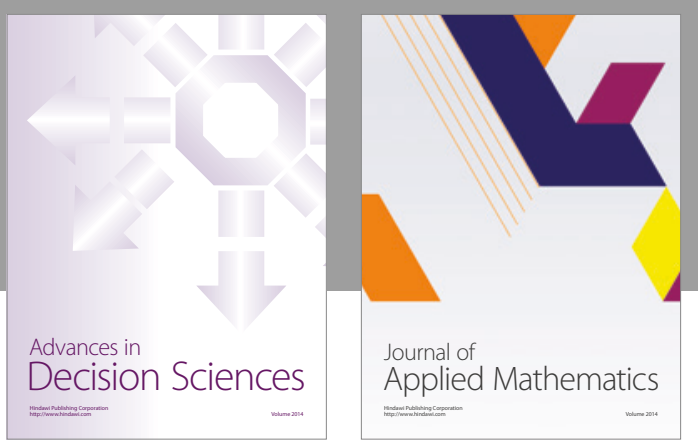

Algebra

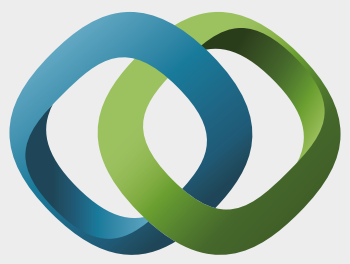

\section{Hindawi}

Submit your manuscripts at

https://www.hindawi.com
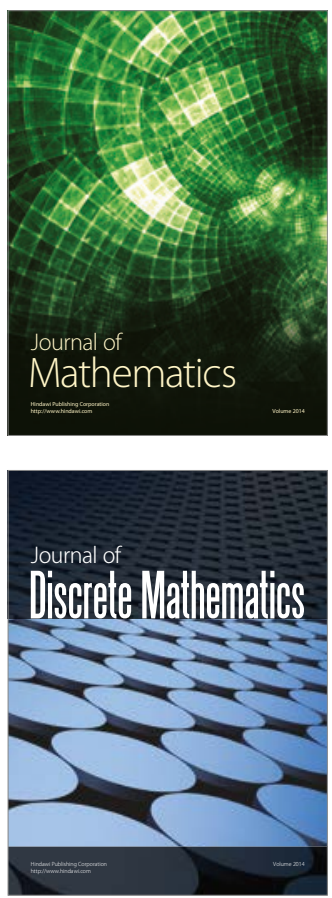

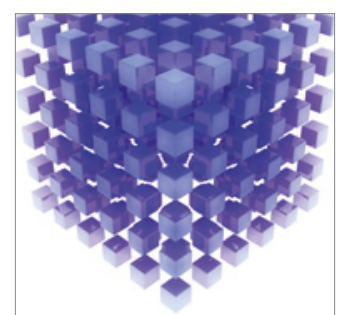

Mathematical Problems in Engineering
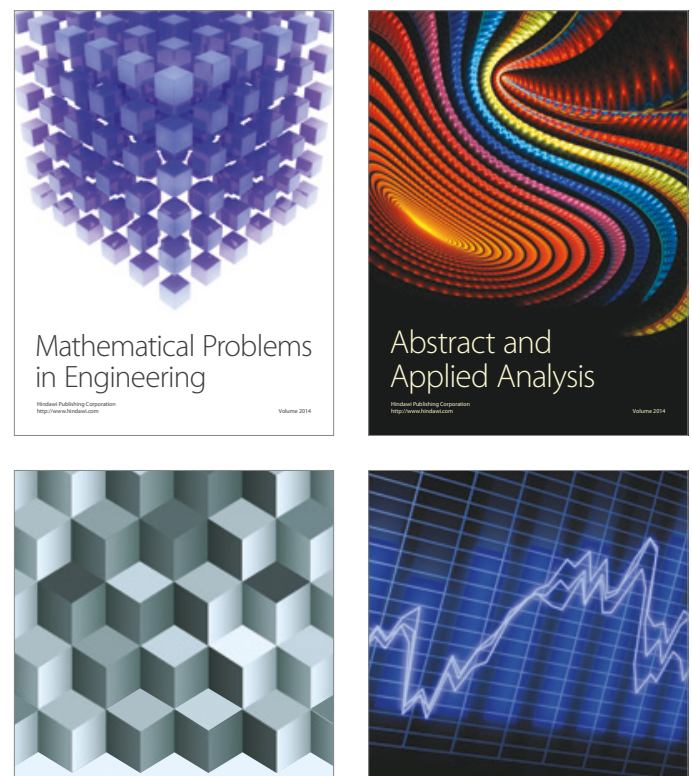

Journal of

Function Spaces

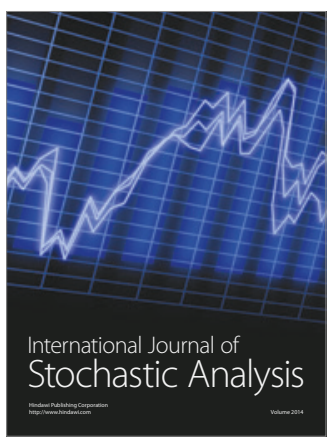

Probability and Statistics
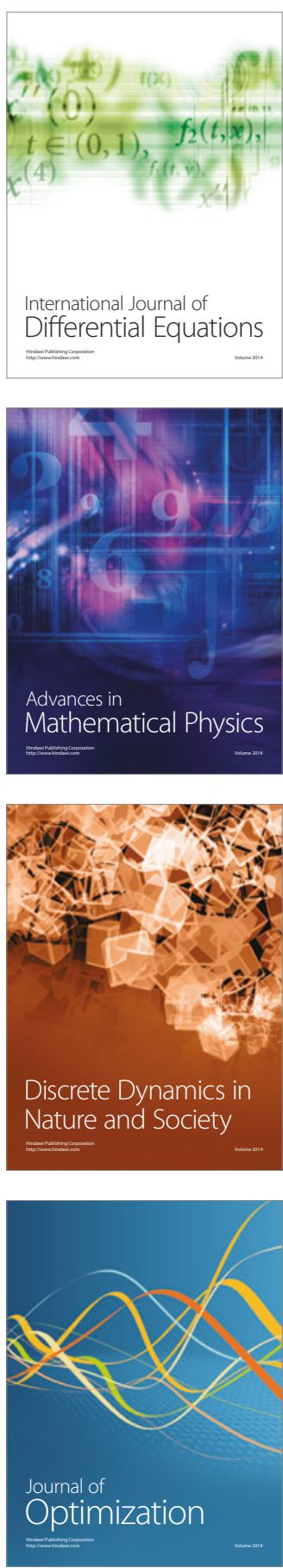